\title{
Nucleotide Sequence Read
}

National Cancer Institute

\section{Source}

National Cancer Institute. Nucleotide Sequence Read. NCI Thesaurus. Code C153349.

The manual or automated determination of the nucleotide order in a nucleic acid fragment obtained after the completion of a sequencing process. 